\title{
EDITORIAL
}

\section{Photonic crystal and topological photonics provide new modulation degrees of freedom}

\author{
Xiaoyong HU (ه) \\ 1 State Key Laboratory for Mesoscopic Physics \& Department of Physics, Collaborative Innovation Center of Quantum Matter, Beijing Academy of \\ Quantum Information Sciences, Nano-optoelectronics Frontier Center of Ministry of Education, Peking University, Beijing 100871, China \\ 2 Collaborative Innovation Center of Extreme Optics, Shanxi University, Taiyuan 030006, China
}

(C) Higher Education Press and Springer-Verlag GmbH Germany, part of Springer Nature 2020

Photonic crystals offer a platform for manipulating light at the mesoscopic scale owing to the unique photonic bandgap properties originating from spatially periodic dielectric distributions. Not only various integrated photonic devices have been realized based on photonic crystals, such as photonic crystal laser and logic devices, but also many physical effects including negative refractive and optical cloak have been realized in photonic crystals. Based on the photonic band structure, topological photonics has been an emerging research hotspot nowadays. Topological photonics provide two new modulation degrees of freedom, i.e., topological state degrees of freedom and energy valley degrees of freedom. It can be expected that topological photonics will not only boost the fundamental study of physical effects and phenomena, but also improve the research of high-performance photonic devices.

Following the research trend in the field of photonics, the journal of Frontiers of Optoelectronics produces a special issue on Photonic Crystal and Topological Photonics in order to promote the research in the area of topological photonics and development of photonic devices based on topological features. In this special issue, Prof. Chan from The Hong Kong University of Science and Technology gives an in-depth comment on the development and research direction of photonic crystal and topological photonics. There are also three research articles and three review articles in this special issue. This special issue will improve the fundamental and application research of the field of photonic crystal and topological photonics.

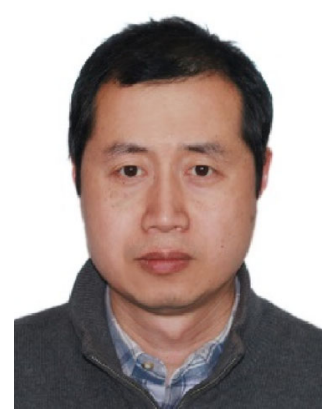

Xiaoyong Hu is a Cheung Kong professor of physics at Peking University. Prof. Hu's current research interests include photonic crystals, plasmonics, topological photonics and integrated photonic devices.

Received March 18, 2020

E-mail: xiaoyonghu@pku.edu.cn 